\title{
Bispectra and Cross-Bispectra of Temperature, Salinity, Sound Velocity and Density Fluctuations with Depth off Northeastern Japan ${ }^{1}$
}

\author{
Gunnar I. Roden and Donna J. Bendiner \\ Depl. of Oceanography, University of Washington, Seattle 98195
}

(Manuscript received 28 July 1972, in revised form 15 January 1973)

\begin{abstract}
Off northeastern Japan, depth profiles of temperature, salinity, sound velocity and density show considerable structure between 30 and $240 \mathrm{~m}$. The structure is asymmetric with respect to the mean and the amplitudes are 10 times larger here than in similar latitudes in the mid-Pacific. By partitioning the observed series in to mean and perturbation series, it is possible to compute the bispectrum and the bicoherence for each variable, as well as the cross-bispectrum and the cross-bicoherence for different variables. The computations were carried out for the wavenumber plane bounded by $\left|k_{1}\right| \leqslant 167$ cycles per kilometer (cpkm), $\left|k_{2}\right| \leqslant 167 \mathrm{cpkm},\left|k_{1}+k_{2}\right| \leqslant 167 \mathrm{cpkm}$, with an areal resolution of $48.2(\mathrm{cpkm})^{2}$.

The "third-power density" of the bispectra decreases toward the Nyquist wavenumber by about three orders of magnitude. The rate of decrease is not constant, but depends upon both wavenumber and direction. There is no statistically significant structure superimposed upon the slopes. The absence of prominent structural features suggests that the bispectra arise from continuum interactions rather than from line interactions. Significant bicoherence is limited to wavenumber pairs that sum to less than $40 \mathrm{cpkm}$. Significant cross-bicoherence for the sound velocity-temperature-salinity perturbation triplet occurs at low and intermediate wavenumbers.
\end{abstract}

\section{Introduction}

In March and April, 1971, the R. V. Thomas G. Thompson made a detailed study of the thermohaline structure in the northwestern Pacific. The structure was particularly pronounced off northeastern Japan, near the boundary of the warm and high salinity Kuroshio with the cold and low salinity Oyashio. Numerous temperature and salinity inversions were observed in the vertical, with scales ranging from a few meters to a few hundred meters. At a given station, the temperature structure was related to the salinity structure in such a way that the resulting density structure was neutral or stable in almost all cases; only near the sea surface did small instabilities occasionally occur. Among neighboring stations, large-scale structural features were usually coherent, while small-scale ones were not.

This paper deals with vertical structural features between 6 and about $60 \mathrm{~m}$, that is, small- and intermediate-scale perturbations from the mean profiles of temperature, salinity, sound velocity [computed from Wilson's equation (Vigoureux and Hersey, 1962)] and $\sigma_{t}$-density. The question arises as to the spectral and bispectral properties of these perturbations and their coherence and bicoherence.

The ordinary spectra of oceanic variables have been investigated in some detail by Grant et al. (1962), Garrett and Munk (1971), Gregg and Cox (1971),

${ }^{1}$ Contribution No. 696 from the Department of Oceanography, University of Washington.
Phillips (1971) and Roden (1971). The observed spectra show that the variance per unit bandwidth decreases with increasing wavenumber according to a power law with variable decay coefficient. In the low-wavenumber region, where buoyancy forces are important, the decay coefficient of the observed spectra is close to $-8 / 3$. In the high-wavenumber region, the decay coefficient of the observed spectra approaches $-5 / 3$. These findings are in agreement with the theory of turbulence in a stratified medium (Phillips, 1966; Charney, 1971) as well as with the theory of wave motion in a sharply layered fluid (Garrett and Munk, 1971). The former requires the decay coefficient to lie between -3 and $-5 / 3$, decreasing with increasing wavenumber, and the latter requires a decay coefficient of -2 . At present, it is difficult to distinguish between the two mechanisms in the intermediate- and high-wavenumber range, because the scatter of points in the observed spectra is such that decay coefficients cannot be determined with much accuracy.

The use of ordinary spectra for extracting information from geophysical series has basic limitations (Tukey, 1965). From the turbulence point of view, it assumes that the process under investigation is fully described by correlation functions of the second order, such as in the Gaussian isotropic case. From the point of view of wave theory, it assumes that the observed fluctuations consist of a linear superposition of independent individual wavelets of random phase, such that the wavelets can pass through each other without distorting amplitude or wavenumber. 
In the ocean, conditions for isotropy rarely exist at low and intermediate wavenumbers, because of horizontal and vertical stratification and the presence of currents. Third and higher order correlation functions are then required to describe the turbulent process (Monin and Yaglom, 1965; Van Atta and Chen, 1970; Paquin and Pond, 1971). Third-order correlation functions and their Fourier transforms, called bispectra, are required also in the study of wave interactions (Hasselmann et al., 1963; Barnett et al., 1971; Huber et al., 1971).

The use of bicorrelations and bispectra in geophysics is just beginning. Hasselmann et al. investigated bispectra of ocean waves in shallow water and found a pleasing agreement between theory and observation. MacDonald (1965) studied the bispectrum of atmospheric pressure records and found strong interaction of the annual peak with itself in some regions of the world. Munk et al. (1965) investigated tidal cusps in the sea level spectrum and attributed them to interaction between tidal lines and the continuum. All the above investigations dealt with the bispectrum of a single variable only. To the authors' knowledge, the cross-bispectrum among different variables has not yet been determined.

The purpose of the present paper is to give a brief summary of the properties of the bicorrelations and bispectra and then to discuss the findings from the region off northeastern Japan.

\section{Theoretical aspects}

\section{a. Cross-bicorrelation}

Let $x(t), y(t)$ and $z(t)$ be three stationary series with respect to time or distance. The cross-bicorrelation, $\varphi_{x y z}$, is then defined as the expectation $E$ of the third moment,

$$
\varphi_{x y z}\left(\tau_{1}, \tau_{2}\right)=E\left[x(l) y\left(t+\tau_{1}\right) z\left(t+\tau_{2}\right)\right],
$$

where $\tau_{1}$ and $\tau_{2}$ are lags. For a finite series of $N$ points, (1) is defined in the region

$$
\left|\tau_{1}\right| \leqslant N ;\left|\tau_{2}\right| \leqslant N ;\left|\tau_{1}+\tau_{2}\right| \leqslant N .
$$

Because the series are assumed stationary, the following relationships exist among the various combinations of the order in which the series are placed:

$$
\varphi_{x y z}\left(\tau_{1}, \tau_{2}\right)=\left\{\begin{array}{l}
\varphi_{x z y}\left(\tau_{2}, \tau_{1}\right) \\
\varphi_{y x z}\left(-\tau_{1}, \tau_{2}-\tau_{1}\right) \\
\varphi_{y z x}\left(\tau_{2}-\tau_{1},-\tau_{1}\right) \\
\varphi_{z x y}\left(-\tau_{2}, \tau_{1}-\tau_{2}\right) \\
\varphi_{z y x}\left(\tau_{1}-\tau_{2},-\tau_{2}\right)
\end{array}\right.
$$

Thus, for a given sequence of three different series, the cross-bicorrelation is asymmetric. If any two of the three series are equal, a twofold symmetry exists in the $\tau_{1}, \tau_{2}$ plane. If all three series are equal, then a sixfold symmetry is observed in this plane.
From a physical point of view, the cross-bicorrelation expresses a double delayed action effect. It measures the joint interaction between three sets of variables displaced relative to each other and gives an indication of the persistence of the interaction.

In fact, (1) can be expressed in terms of a third-order probability density (Lee, 1963),

$$
\varphi_{x y z}\left(\tau_{1}, \tau_{2}\right)=\iint_{-\infty}^{\infty} \int p\left[x\left(t_{1}\right) y\left(t_{2}\right) z\left(l_{3}\right) ; \tau_{1}, \tau_{2}\right],
$$

where $p$ is the probability that: $x\left(t_{1}\right)$ lies between $x$ and $d x ; y\left(t_{2}\right)$ lies between $y$ and $d y$; and $z\left(t_{3}\right)$ lies between $z$ and $d z$.

For a jointly Gaussian process, the cross-bicorrelation is zero. A nonvanishing cross-bicorrelation gives, therefore, a first indication that the random process is of an order higher than two.

\section{b. Cross-bispectrum}

The cross-bispectrum $S$ is the Fourier transform of a suitably smoothed cross-bicorrelation,

$$
\begin{array}{r}
S_{x y z}\left(k_{1}, k_{2}\right)=(\Delta l)^{2} \sum_{\tau_{1}=-m}^{m-1} \sum_{\tau_{2}=-m}^{m-1} \varphi_{x y z}\left(\tau_{1}, \tau_{2}\right) \psi_{s}\left(\tau_{1}, \tau_{2}\right) \\
\times \exp \left[-\frac{\pi}{m}\left(k_{1} \tau_{1}+k_{2} \tau_{2}\right)\right],
\end{array}
$$

where $k_{1}$ and $k_{2}$ refer to frequency or wavenumber, $m$ is the maximum number of the spectral estimates and lags, $\Delta l$ is the sampling interval, and $\psi_{s}$ is a bicorrelation smoothing function such as

$\psi_{s}=\frac{1}{8}\left(1+\cos \frac{\pi \tau_{1}}{m}\right)\left(1+\cos \frac{\pi \tau_{2}}{m}\right)\left[1+\cos \frac{\pi}{m}\left(\tau_{1}-\tau_{2}\right)\right]$.

The smoothing operation is necessary in order to obtain bispectral estimates that possess desirable sampling characteristics (Godfrey, 1965; Rosenblatt and Van Ness, 1965). The units of (4) are amplitude cubed per unit area of resolution (two-dimensional bandwidth).

The cross-bispectrum of a real stationary process is a complex quantity determined in the half plane, because $S\left(-k_{1},-k_{2}\right)=S^{*}\left(k_{1}, k_{2}\right)$, where the asterisk refers to the complex conjugate. In order to avoid aliasing, the cross-bispectrum must be small outside the region bounded by the Nyquist frequency $k_{n}$, that is, outside $\left|k_{1}\right| \leqslant k_{n},\left|k_{2}\right| \leqslant k_{n}$ and $\left|k_{1}+k_{2}\right| \leqslant k_{n}$.

The following relations hold among the crossbispectra when the order of the series is interchanged:

$$
S_{x y z}\left(k_{1}, k_{2}\right)=\left\{\begin{array}{l}
S_{x z y}\left(k_{2}, k_{11}\right) \\
S_{y x z}\left(-k_{1}-k_{2}, k_{2}\right) \\
S_{y z x}\left(-k_{1}-k_{2}, k_{1}\right) \\
S_{z x y}\left(k_{2},-k_{1}-k_{2}\right) \\
S_{z y x}\left(k_{1},-k_{1}-k_{2}\right)
\end{array}\right.
$$


Thus, when all three series are different, the crossbispectrum is uniquely determined in the half plane. If any two of the three series are equal, a twofold symmetry occurs in this plane. If all three series are equal, a sixfold symmetry is observed and the bispectrum is determined, for example, by a triangular region in the first quadrant, with the abscissa as the base and the point $\left(\frac{1}{2} m, \frac{1}{2} m\right)$ as the apex.

\section{c. Cross-bicoherence as a measure of skewness}

While the cross-bispectrum alone contains sufficient information about the third moment one wishes to study, this information may be more easily interpreted if certain functions of the cross-bispectrum are studied.

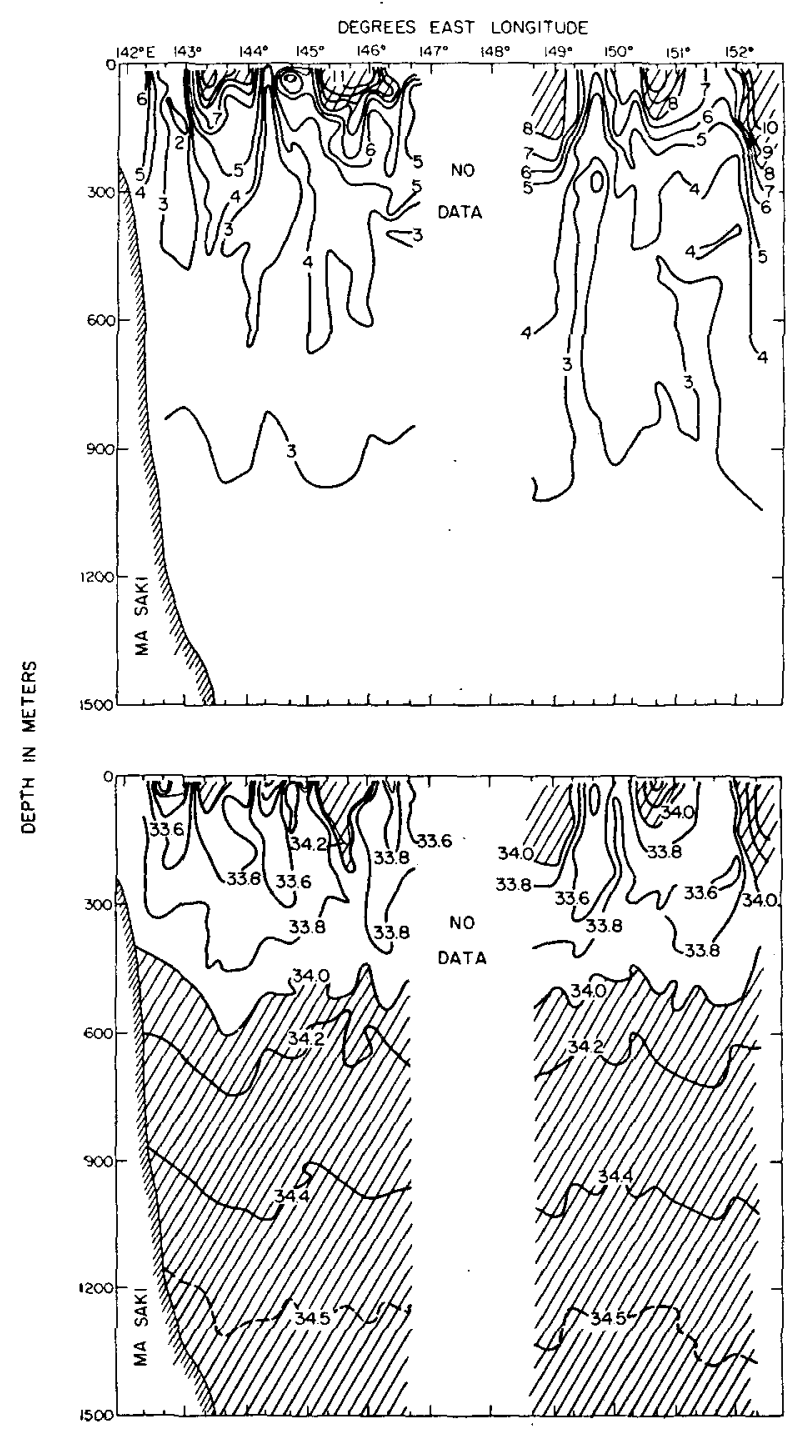

FIG. 1. Vertical distribution of temperature (top) and salinity (bottom) off northeastern Japan. The section extends eastward along latitude $40 \mathrm{~N}$ and was occupied between 26 and 30 April 1971. Temperatures $>8 \mathrm{C}$ and salinities $>34.0 \%$ are crosshatched to indicate water of Kuroshio origin.
One such function is obtained by expressing the crossbispectrum in polar coordinates and relating it to the triple product of the ordinary spectra, $S_{x x}, S_{y y}, S_{z z}$, in a manner reminiscent of the determination of the ordinary skewness coefficient. The result is

$$
\begin{aligned}
S_{x y z}\left(k_{1}, k_{2}\right) & =\left\{\frac{\left|S_{x y z}\left(k_{1}, k_{2}\right)\right|}{\left[S_{x x}\left(k_{1}\right) S_{y y}\left(k_{2}\right) S_{z z}\left(k_{3}\right)\right]^{\frac{1}{z}}}\right\} \\
\times & {\left[S_{x x}\left(k_{1}\right) S_{y y}\left(k_{2}\right) S_{z z}\left(k_{3}\right)\right]^{\frac{1}{2}} \exp \left[i \theta\left(k_{1}, k_{2}\right)\right], }
\end{aligned}
$$

where the term in braces is called the bispectral skewness (Hinich and Clay, 1968) or, somewhat less descriptively, the bicoherence (Hasselmann et al., 1963). The phase angle $\theta$ is called the biphase. The term cross-bicoherence and cross-biphase have not appeared in the literature so far, but will be used here when discussing relationships among different variables.

The cross-bicoherence is, by definition, a real-valued quantity equal to or larger than zero. In the special case that the nonlinear process is due to first-order multiplicative interaction between the major frequencies or wavenumbers, such that the component at $k_{1}+k_{2}$ is equal or proportional to the product of the components at $k_{1}$ and $k_{2}$, the cross-bicoherence is unity. It follows, therefore, that if the cross-bicoherence differs from unity significantly, the nonlinear process cannot be of the above simple type. Thus, the cross-bicoherence can be used to discriminate between types of nonlinear processes.

\section{Field measurements off northeastern Japan \\ a. Instrumentation and data treatment}

The thermohaline structure was measured with the Bissett-Berman electronic salinity-temperature-depth sensor, commonly known as the STD. This instrument has a temperature accuracy of $0.01 \mathrm{C}$, a salinity accuracy of $0.03 \%$, and a depth accuracy of $0.25 \%$ of the actual depth. The STD was lowered from the surface to $1500 \mathrm{~m}$ at a rate of $90 \mathrm{~m} \mathrm{~min}^{-1}$, yielding data every $3 \mathrm{~m}$, on the average. The digitized data were passed through an error-screening routine and through a linear interpolation scheme to yield values at exactly $3 \mathrm{~m}$ intervals.

For purposes of further analysis, the equally-spaced series were partitioned into mean and perturbation series. The mean series was obtained by smoothing the original. series with a 41-point binomial weighting array, with the weights given by

$$
w(i)=\left[\frac{m !}{(m-i) ! i !}\right]^{p} / \sum_{i}\left[\frac{m !}{(m-i) ! i !}\right]^{p},
$$

where $m=40$ and $p=0.5$ (Roden, 1971). The perturbation series was obtained by subtracting the mean series from the original series. This method of high-pass 
filtering has the response

$$
Y(k)=1-\sum_{i} w(i) \cos (2 \pi k \Delta l)
$$

where $k$ is a wavenumber and $\Delta l$ the sampling interval here taken as $0.003 \mathrm{~km}$. The spectral and bispectral transfer functions of this high-pass filter are $Y^{2}(k)$ and $Y\left(k_{1}\right) Y\left(k_{2}\right) Y\left(k_{1}+k_{2}\right)$, respectively.

\section{b. General thermohaline environment off northeastern Japan}

Before discussing the fine-structure, it is of interest to look briefly at the main large-scale features of the vertical temperature and salinity distributions. These are shown in Fig. 1 for a section along latitude $40 \mathrm{~N}$. The section lies close to the northern boundary of the Kuroshio and is marked by numerous intrusions of warm and high-salinity water in the upper layers. The intrusions are about $60-80 \mathrm{~km}$ wide, extend downward to about $200 \mathrm{~m}$ and are characterized by temperatures above $8 \mathrm{C}$ and salinities above $34.0 \%$. Surrounding the warm and saline intrusions is water of subarctic origin, characterized by low temperatures and by low salinities in the upper $400 \mathrm{~m}$. Within the subarctic water mass, there are cores of very cold water, where temperatures vary between 0 and $2 \mathrm{C}$; these occur usually in the depth range from 100 to $300 \mathrm{~m}$ and are of limited vertical extent (only the two largest ones are shown in the figure). The very cold water originates in the sea of Okhotsk and is carried southward by the Oyashio.

\section{c. Characteristics of the fine-structure}

Typical examples of temperature, salinity, sound velocity and $\sigma_{t}$-density perturbations, as obtained by binomial filtering, are shown in Fig. 2 for a station located $65 \mathrm{~km}$ due east of Ma Saki Light, Japan. Almost all of the large structural features occur between 30 and $240 \mathrm{~m}$. Near the surface, perturbations are small because of turbulent mixing and diffusion. At great depths, the perturbations are small because of slow currents and weak thermohaline gradients. Hence, a region of increased perturbation amplitudes generally is encountered between the surface and deep layers. This is a very characteristic feature of the oceanic thermohaline structure. What makes the region off northeastern, Japan unique however, is the size of the

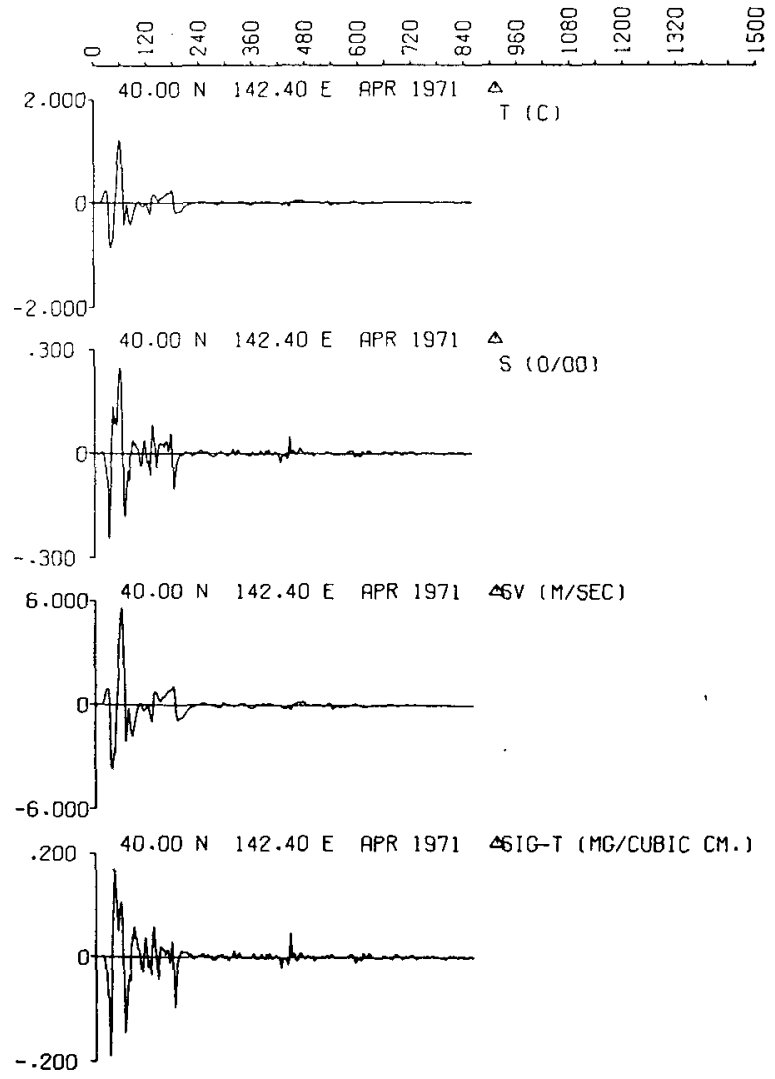

Fig. 2. Perturbations from the mean as a function of depth $(\mathrm{m})$, obtained by binomial filtering with a 41-point array: $T$ temperature, $S$ salinity, SV sound velocity, SIG- $T \sigma_{t}$-density. The station is $65 \mathrm{~km}$ off Ma Saki Light, Japan.

structure. The perturbation amplitudes encountered in this region are about 10 times larger than those encountered at similar latitudes in the central Pacific (Table 1). The large amplitudes in the frontal region off Japan result from the confluence and intermingling of two current systems of different origin. The vertical length scales of the perturbations, as obtained from an analysis of zero-crossing intervals, are given in Table 2 as a function of longitude. For temperature and sound velocity, the average "thickness" of the perturbations varies between 13 and $19 \mathrm{~m}$; for salinity and $\sigma_{t}$-density it varies between 7 and $11 \mathrm{~m}$. The marked difference in the average "thickness" of temperature and salinity perturbations is a noteworthy feature of the oceanic

TARLE 1. Maximum perturbation amplitudes above and below the mean $(>0,<0)$ of temperature $(T)$, salinity $(S)$, sound velocity $(C)$, and $\sigma_{t}$-density in the northwestern and central Pacific.

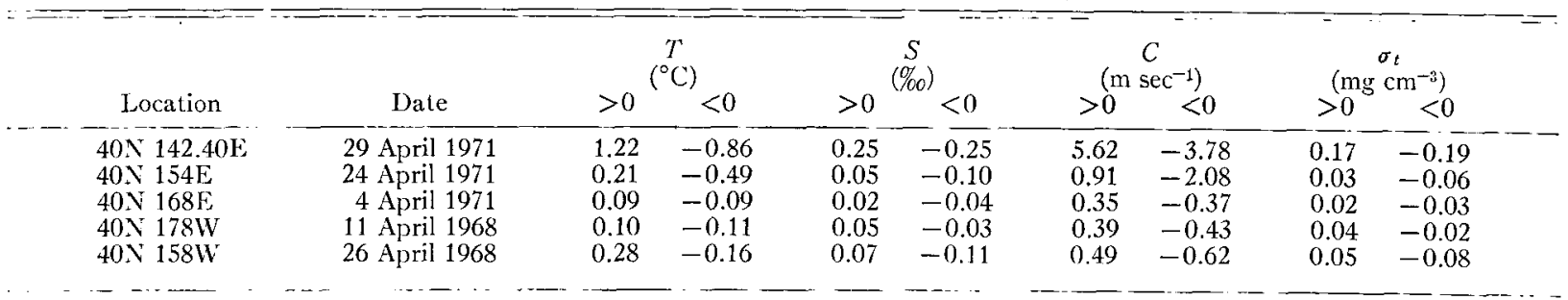


TABLE 2. Average vertical length scales $(\mathrm{m})$ of perturbations above and below the mean $(>0,<0)$ for temperature $(T)$, salinity $(S)$, sound velocity $(C)$, and $\sigma_{t}$-density in the northwestern and central Pacific.

\begin{tabular}{|c|c|c|c|c|c|c|c|c|c|}
\hline \multirow[b]{2}{*}{ Location } & \multirow[b]{2}{*}{ Date } & \multicolumn{2}{|c|}{$\begin{array}{c}T \\
\left({ }^{\circ} \mathrm{C}\right)\end{array}$} & \multicolumn{2}{|c|}{$\underset{(\%)}{S}$} & \multicolumn{2}{|c|}{$\begin{array}{c}C \\
(\mathrm{~m} \mathrm{sec}-1)\end{array}$} & \multicolumn{2}{|c|}{$\begin{array}{c}\sigma_{t} \\
\left(\mathrm{mg} \mathrm{cm}^{-3}\right)\end{array}$} \\
\hline & & $>0$ & $<0$ & $>0$ & $<0$ & $>0$ & $<0$ & $>0$ & $<0$ \\
\hline $40 \mathrm{~N} 142.40 \mathrm{E}$ & 29 April 1971 & 18.1 & 19.2 & 9.3 & 11.2 & 17.9 & 18.5 & 9.8 & 10.9 \\
\hline $40 \mathrm{~N} 154 \mathrm{E}$ & 24 April 1971 & 15.8 & 13.0 & 9.7 & 7.8 & 15.9 & 13.5 & 8.6 & 7.5 \\
\hline $40 N 168 E$ & 4 April 1971 & 16.7 & 16.7 & 9.6 & 7.0 & 16.2 & 16.4 & 9.7 & 7.9 \\
\hline $40 \mathrm{~N} 178 \mathrm{~W}$ & 11 April 1968 & 16.7 & 15.2 & 9.0 & 8.8 & 17.4 & 15.9 & 9.5 & 9.9 \\
\hline $40 \mathrm{~N} 158 \mathrm{~W}$ & 26 April 1968 & 16.2 & 16.1 & 10.7 & 10.6 & 16.9 & 16.0 & 11.8 & 11.1 \\
\hline
\end{tabular}

thermohaline structure, which may be related to the difference in turbulent diffusion rates for heat and salt.

The perturbation series, such as shown in Fig. 2, are asymmetrical with respect to the mean, indicating a nonvanishing skewness coefficient and a nonvanishing bicorrelation. For the station off Ma Saki Light, the skewness coefficients are 1.69 for temperature, 0.48 for salinity, 2.00 for sound velocity, and -0.57 for $\sigma_{t}$-density.

The normalized bicorrelations for the perturbations illustrated in Fig. 2 are shown in Fig. 3 for lag lengths up to $72 \mathrm{~m}$. Normalization was achieved by dividing the bicorrelation by its maximum value, making it

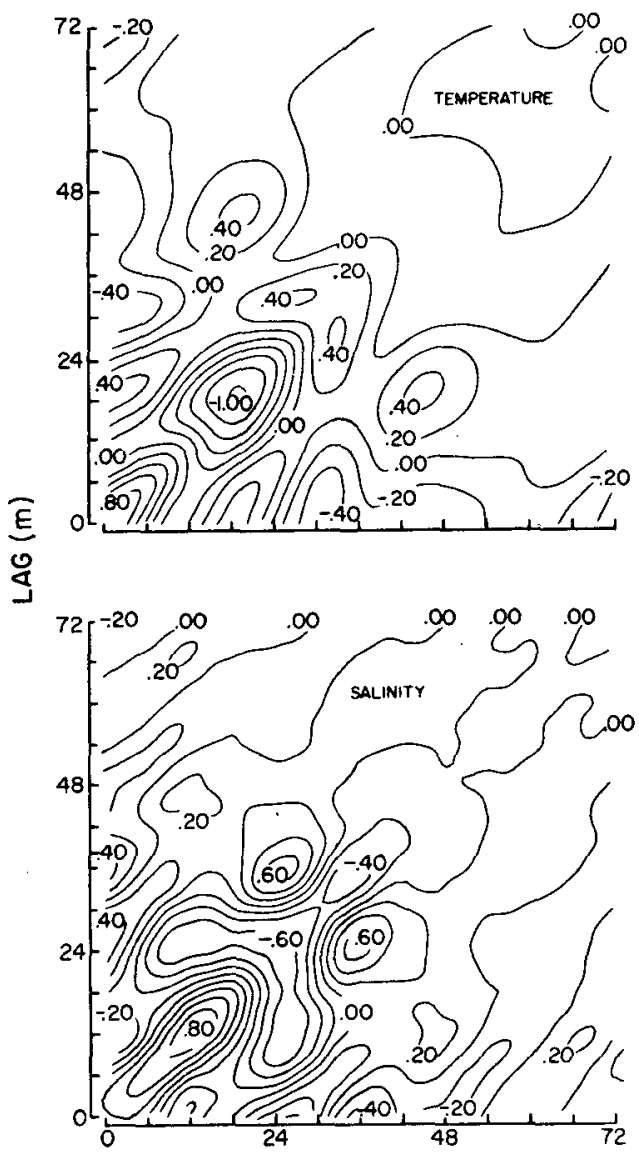

dimensionless. The normalized bicorrelation thus ranges between -1 and +1 and, for a single variable, is symmetric with respect to the diagonal through the origin. The exact confidence limits for the bicorrelation have not yet been determined. For a nearly normal distribution, the $95 \%$ confidence limits would be \pm 0.2 , approximately (Kendall and Stuart, 1958).

Numerous topographic features occur in the low lag-length region of the bicorrelation. The size and shape of the rises and depressions give an indication of the correlation scales involved. If the perturbations at a given depth were uncorrelated with those occuring at two other depths, the bicorrelation would consist of a
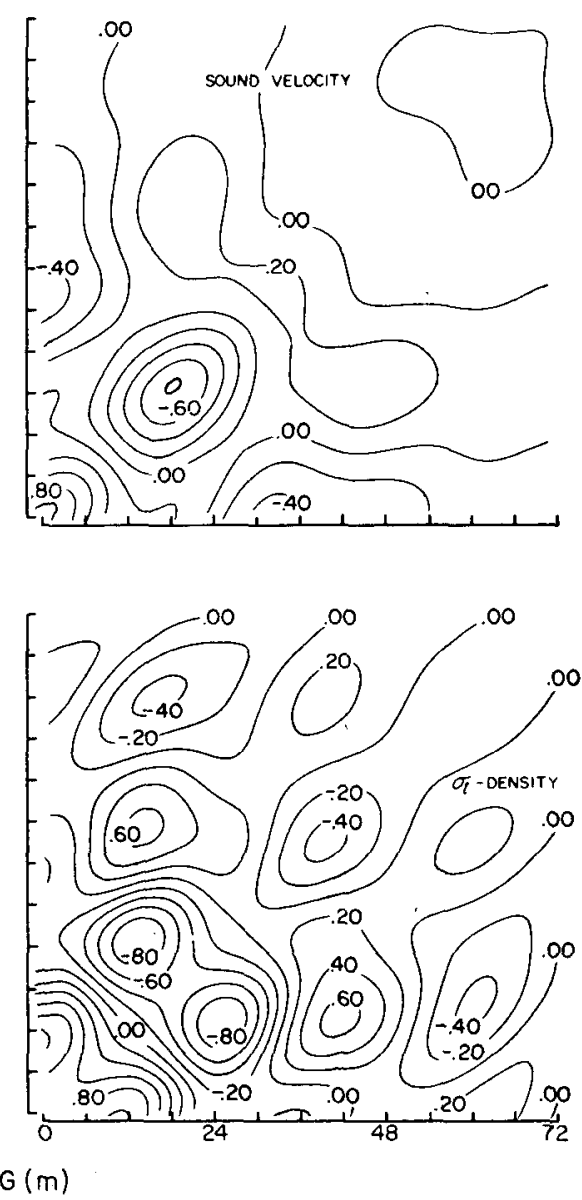

FIG. 3. Normalized bicorrelation of temperature, salinity, sound velocity, and $\sigma_{\ell}$-density perturbations as a function of lag length. 


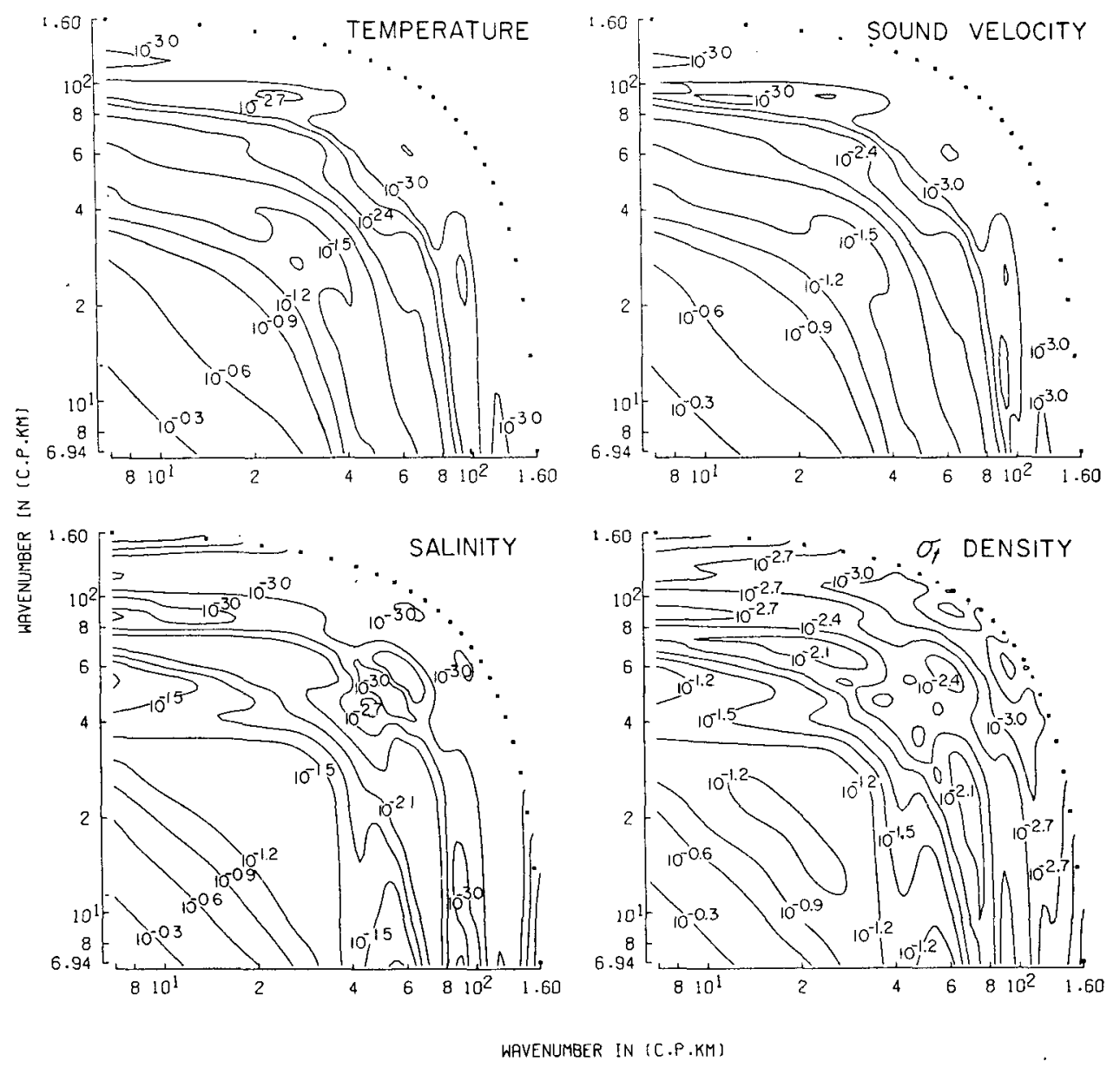

FIG. 4. Normalized bispectra of temperature, salinity, sound velocity, and $\sigma_{t}$-density, corrected for binomial filtering. The dotted line refers to the Nyquist wavenumber.

single peak centered at the origin and the correlation scales would be less than the sampling interval. If the perturbations at a given depth were correlated with the square of those occurring at one other depth, the bicorrelation would consist of a single topographic feature centered along the $45^{\circ}$ line through the origin, and the correlation scales could be determined from the lengths the bicorrelation remained above 0.2 and below -0.2 . In actuality, conditions are more complicated. It is seen from Fig. 3 that the perturbations at a given point are related to those occurring at two points for separation distances up to about $50 \mathrm{~m}$. The elongated shapes of the rises and depressions indicate that the correlation scales are highly anisotropic in the lag-length plane.

\section{Wavenumber bispectra of the observed vertical temperature, salinity, sound velocity, and $\sigma_{t^{-}}$ density structure}

\section{a. Ordinary bispectra with respect to depth}

The normalized magnitudes for the bispectra of temperature, salinity, sound velocity, and $\sigma_{t}$-density are shown in Fig. 4 on a triple logarithmic scale. The computations were carried out in the wavenumber plane boúnded by $\left|k_{1}\right| \leqslant 167 \mathrm{cpkm},\left|k_{2}\right| \leqslant 167 \mathrm{cpkm}$, $\left|k_{1}+k_{2}\right| \leqslant 167 \mathrm{cpkm}$, with an areal resolution of 48.2 $(\mathrm{cpkm})^{2}$. All bispectra were corrected for binomial filtering by dividing them by the bispectral transfer function derived from (8). It must be kept in mind that the bispectral estimates at wavenumbers summing to less than $15 \mathrm{cpkm}$ are somewhat arbitrary, because the shortness of the original record does not allow for many realizations of large-scale fluctuations. Normalization of the bispectrum was achieved by dividing its magnitude by the maximum value. The resulting expressions are dimensionless and facilitate the comparison of bispectra for different variables. The dotted line refers to the Nyquist wavenumber. In accordance with (6) the bispectra for a single variable are symmetric with respect to the diagonal through the origin.

The outstanding feature in Fig. 4 is the rather smooth decrease of the "third-power density" from the origin toward the Nyquist wavenumber. The total decrease is about three orders of magnitude. The rate of decrease is not constant, however, but depends upon both wave- 


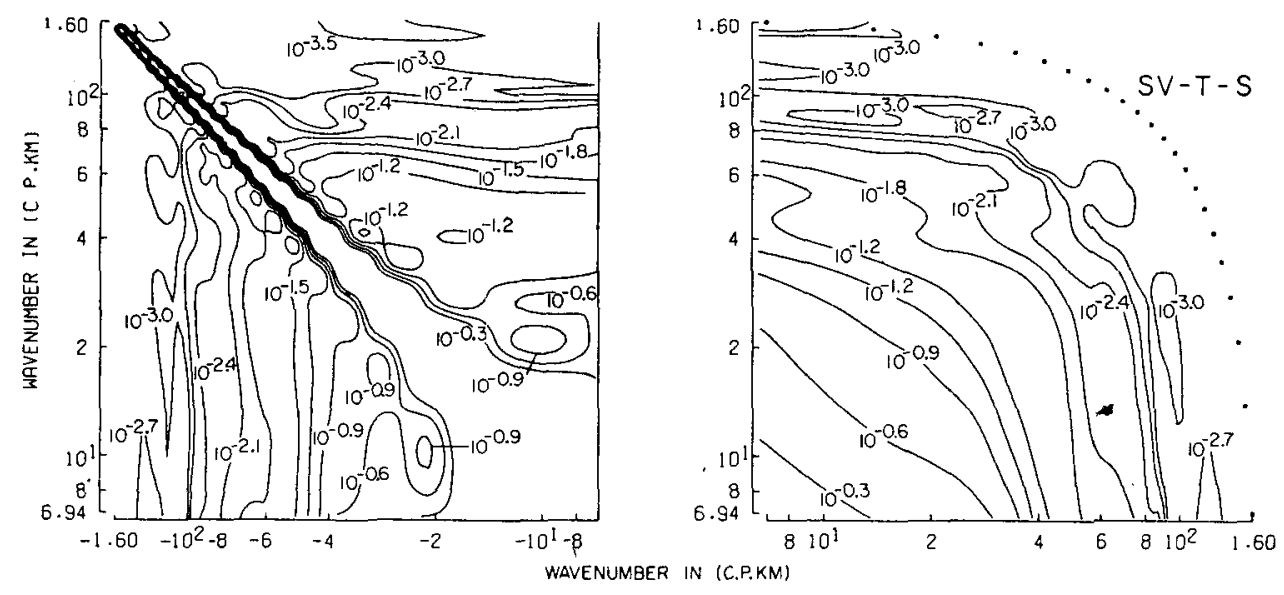

FIG. 5. Normalized cross-bispectrum for the triplet sound velocity-temperature-salinity, corrected for binomial filtering. The dotted line refers to the Nyquist wavenumber.

number and direction, indicating anisotropic conditions. The anisotropy has a tendency to decrease toward the Nyquist wavenumber. The decay coefficients for the "third-power density" vary between -1 and -8 , being largest where the sum of the wavenumber lies between 60 and $100 \mathrm{cpkm}$. In the high-wavenumber region, there are several rises and depressions superimposed upon the slopes. Since none of them depart much from the surrounding terrain, they are probably insignificant from a statistical point of view.

The absence of significant rises and depressions in the bispectra suggests that they result from the interaction of a great many perturbation scales and not just a few ; in other words, continuum-continuum-continuum interactions dominate over line-line-line interactions. The bispectra described above could thus arise from turbulent interactions or from interactions within a random field of internal waves, or both. At present, with no theoretical guidelines, it is impossible to differentiate between the two processes and to assess the relative importance of each. The fact that the bispectra rise toward where the sum of the wavenumbers approaches zero rules out white noise-type interactions among the continua (in which the bispectra would be constant); rather, the fact is suggestive of interactions among the perturbations and the main thermohaline structure.

\section{b. Cross-bispectrum of the triplet sound velocity- temperature-salinity}

The normalized magnitude of the cross-bispectrum with respect to depth for this triplet is shown in Fig. 5 on a triple logarithmic scale. The dotted line refers to the Nyquist wavenumber. When no filtering is applied to the original series, the cross-bispectrum is defined in the upper wavenumber half-plane. With filtering, difficulties are encountered, where the filter transfer function is zero, because the corrected cross-bispectrum would approach infinity there. The transfer function of the high-pass filter used is zero along the axes, and along the diagonal in the second quadrant. Hence, the corrected cross-bispectrum shown in Fig. 5 is not defined along these lines. It should also be noted that the crossbispectrum estimates for the regions where the wavenumbers sum to less than $15 \mathrm{cpkm}$ are crude, because of the shortness of the original series.

The outstanding feature of the cross-bispectrum for the triplet is the simplicity with which the joint "thirdpower density" decreases from the maximum toward the Nyquist wavenumber. The total decrease covers about three decades on the logarithmic scale. The contour lines are almost symmetric with respect to the $45^{\circ}$ line through the origin, which is not surprising, because sound velocity is strongly dependent upon temperature and less so upon salinity. The slopes are essentially monotonic, with only minor rises and depressions superimposed upon them.

The absence of significant rises and depressions in the cross-bispectrum rules out simple line-type interactions among wavenumbers of the three different variables. Rather, the continua of all three variables seem to interact, which points toward the importance of random processes in the ocean.

\section{Wavenumber bicoherence and cross-bicoher- ence of the vertical temperature, salinity, sound velocity, and $\sigma_{t}$-density perturbations}

\section{a. Ordinary bicoherence}

The bicoherence of temperature, salinity, sound velocity, and $\sigma_{i}$-density perturbations is shown in Fig. 6, where the dotted line refers to the Nyquist wavenumber. As was mentioned before, the bicoherence expresses the bispectral distribution of skewness, just like the ordinary coherence may be regarded as an expression for the spectral distribution of the correlation coefficient. While the exact $95 \%$ confidence limits for the bicoherence have not yet been determined, approximate confidence limits can be obtained by considering 


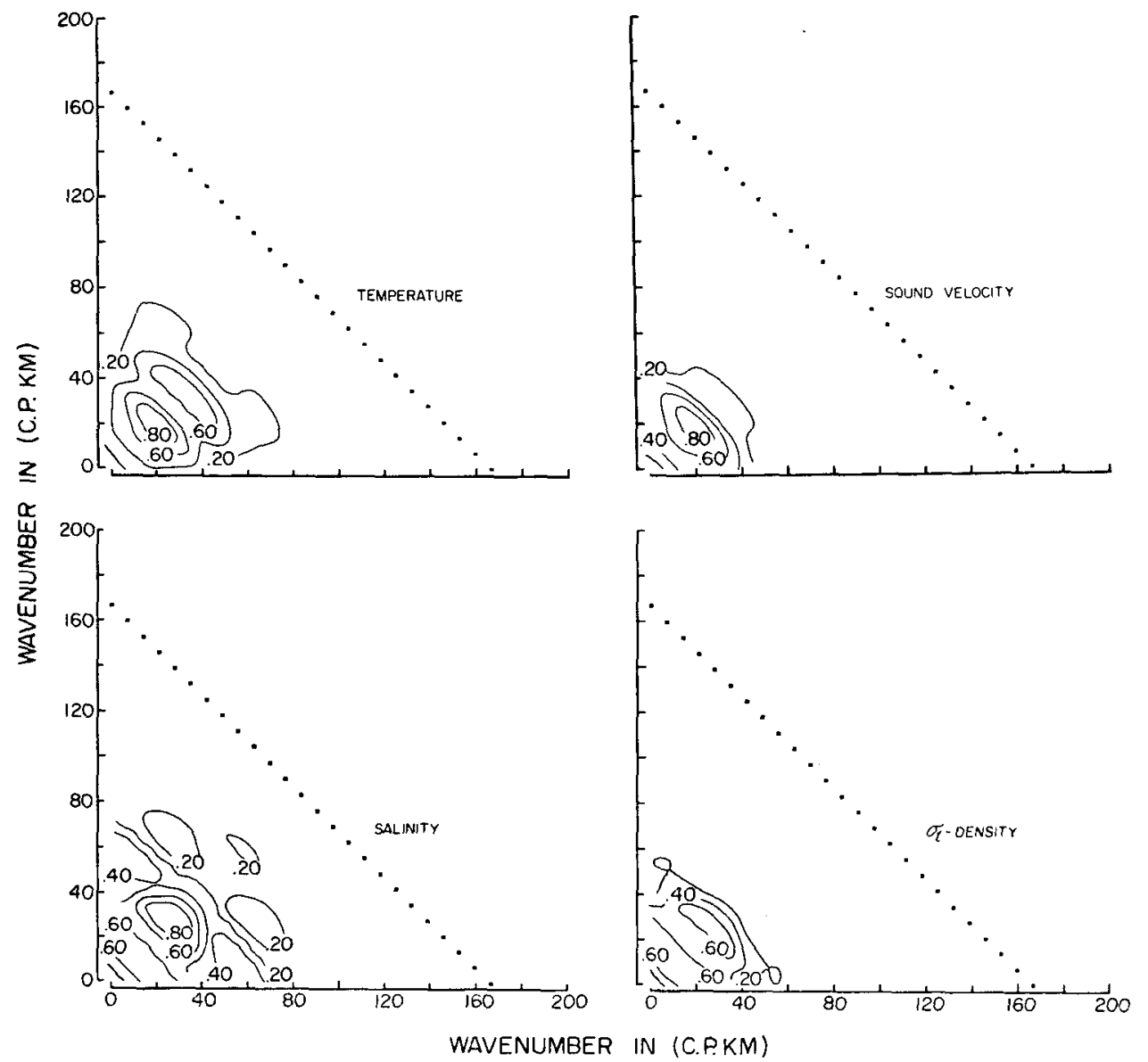

FIG. 6. Bicoherence of temperature, salinity, sound velocity, and $\sigma_{t}$-density perturbations. The dotted line refers to the Nyquist wavenumber.

a nearly normal distribution, for which the standard error of the coefficient of skewness is $(6 / \mathrm{V})^{\frac{1}{2}}$, where $N$ refers to the sample size (Kendall and Stuart, 1958). When $N$ is regarded as the degrees of freedom, this gives also an estimate of the standard error of the bicoherence (MacDonald, 1965). In the present case, with $N=31.8$, the standard error equals 0.43 .

The noteworthy features in Fig. 6 are the peaks and elongated ridges in the region where the wavenumbers sum to less than $80 \mathrm{cpkm}$. The most pronounced peaks tend to occur along a $45^{\circ}$ line from the origin and the ridges are oriented perpendicular to it. Only those above 0.4 should be considered statistically significant. The principal peak of the bicoherence and the wavenumber pair (cpkm) at which they occur are as follows: temperature, 0.95 at $(21,21)$; salinity, 0.98 at $(28,28)$; sound velocity, a single peak of 0.97 at $(21,21)$; and $\sigma_{t}$-density, 0.68 at $(28,28)$. The bicoherence becomes statistically insignificant where the sum of the wavenumbers exceeds $40 \mathrm{cpkm}$.

The physical interpretation is that bispectral skewness is important only in the low and intermediate wavenumber regions, where buoyancy forces are dominant. With increasing wavenumber, the role of the buoyancy forces diminishes and so does that of the bispectral skewness.

\section{b. Cross-bicoherence of the triplet sound velocity- temperature-salinity}

The cross-bicoherence for this perturbation triplet is shown in Fig. 7, where the dotted line refers to the Nyquist wavenumber. The cross-bicoherence is defined inside the region bounded by the dotted line and the abscissa. Estimates for the region, where the wavenumbers sum to less than $15 \mathrm{cpkm}$, are crude because the shortness of the original records does not allow for many realizations of large-scale fluctuations.

The outstanding topographical feature in Fig. 7 is the elongated ridge of high bicoherence in the center of the wavenumber half-plane. The ridge is oriented at an angle of $-45^{\circ}$ and is bounded on the sides by wavenumber pairs that sum to $\pm 40 \mathrm{cpkm}$, approximately. Outside this ridge, the cross-bicoherence is statistically insignificant. The implication is that intermediate- and low-wavenumber perturbations of the sound velocity- 


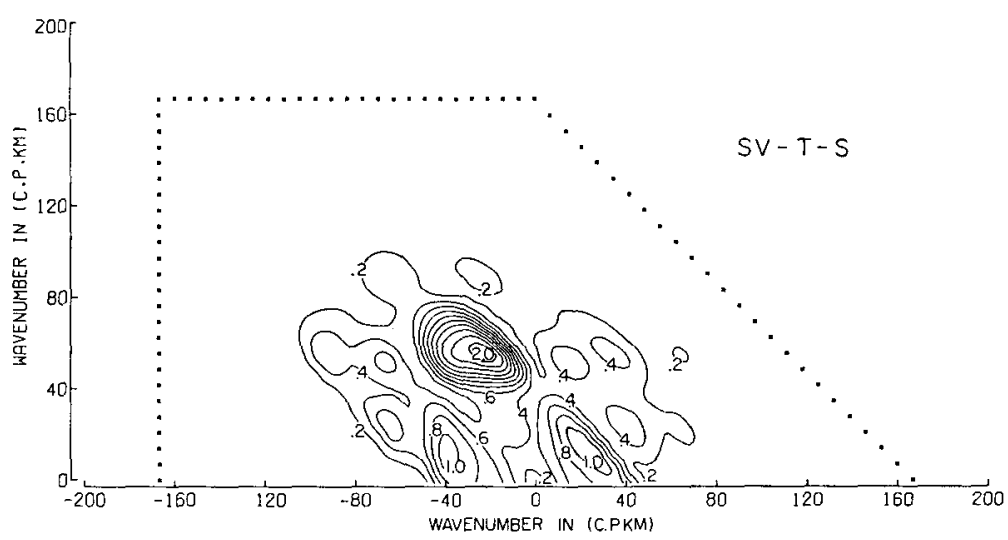

FIG. 7. Cross-bicoherence for the triplet sound velocity-temperature-salinity perturbations. The dotted line refers to the Nyquist wavenumber.

temperature-salinity triplet are correlated, while highwavenumber perturbations are not.

\section{Conclusions}

The following conclusions can be drawn from an analysis of temperature, salinity, sound velocity, and $\sigma_{t}$-density perturbations off northeastern Japan:

1) The perturbations from the mean profiles depend strongly upon depth, with the largest amplitudes occurring between 30 and $240 \mathrm{~m}$.

2) The perturbation amplitudes off northeastern Japan are about 10 times larger than those occurring in similar latitudes in the mid-Pacific.

3) The perturbation series are asymmetric $\cdot$ with respect to the mean, indicating that their description in the wavenumber domain requires knowledge not only of the ordinary spectrum, but also of the bispectrum.

4) The "third-power density" of the bispectra decreases toward the Nyquist wavenumber by about three orders of magnitude. The decrease is not constant, but depends upon both magnitude and direction.

5) There are no statistically significant ridges or depressions superimposed upon the slopes of the bispectra. The absence of prominent structural features suggests that the bispectra arise from continuum interactions rather than from line interactions. Continuum interactions may be related to such processes as nonGaussian turbulence or a non-Gaussian random wave field. The present information is insufficient to distinguish between the different processes.

6) The cross-bispectrum for the sound velocitytemperature-salinity perturbation triplet shows that the joint "third-power density" decreases smoothly, but at nonuniform rates, toward the Nyquist wavenumber. The absence of significant superstructure suggests continuum interactions among the variables.

7) The cross-bicoherence for the sound velocitytemperature-salinity perturbation triplet suggests a significant relationship between the variables at intermediate and low wavenumbers.

Acknowledgments. We are indebted to M. Rattray, C. A. Barnes, W. Bendiner, R. H. Fleming and E. Hasselmann for advice. The scientific and operational crew of the R. V. Thomas G. Thompson, Captain Robert Schelling, commanding, is to be commended for outstanding performance.

The research reported herein was supported by the Office of Naval Research under Contract N-00014-67A-0103-0014, Project 083012.

\section{REFERENCES}

Barnett, T. P., el al., 1971: Bispectrum analysis of electroencephalogram signals during waking and sleeping. Science, 172, 401-403.

Charney, J. G., 1971 : Geostrophic turbulence. J. Atmos. Sci., 28, $1087-1095$.

Garrett, C., and W. H. Munk, 1971 : Internal wave spectra in the presence of fine-structure. J. Phys. Oceanogr., 1, 196-202.

Godfrey, M. D., 1965: An exploratory study of the bi-spectrum of economic time series. Appl. Stalist. (London), 14, 48-69.

Grant, H. L., R. W. Stewart and A. Moilliet, 1962: Turbulence spectra from a tidal channel. J. Fluid Mech., 12, 241-263.

Gregg, M. C., and C. S. Cox, 1971: Measurements of oceanic microstructure of temperature and electrical conductivity. Deep-Sea Res., 18, 925-934.

Hasselmann, K., W. H. Munk and G. MacDonald, 1963 : Bispectra of ocean waves. Symposium on Time Series Analysis, M. Rosenblatt, Ed., New York, Wiley, 125-139.

Hinich, M. J., and C. S. Clay, 1968: The application of the discrete Fourier transform in the estimation of power spectra, coherence and bispectra of geophysical data. Rev. Geoplyys., 6, 347-364.

Huber, P. J., et al., 1971: Statistical methods for investigating phase relations in stationary stochastic processes. IELE Trans. Audio Electroacoustics, 19, 78-86.

Kendall, M. G., and A. Stuart, 1958: The Advanced Theory of Statistics, Vol. 1. London, Griffin, 433 pp.

Lee, Y. W., 1963: Statistical Theory of Communication. New York, Wiley, $509 \mathrm{pp}$. 
MacDonald, G. J. F., 1965: The bispectra of atmospheric pressure records. Proc. IBM Sci. Computing Symposium on Statistics, 21-23 October 1963, New York, IBM Data Processing Division, White Plains, 323 pp.

Monin, A. S., and A. M. Yaglom, 1965: Stalisticheskaya Gidromekhanika, Vol. 1. Moscow, Nauka, 639 pp.

Munk, W. H., B. Zetler and G. W. Groves, 1965: Tidal cusps. Geophys. J., 10, 211-219.

Paquin, J. E, and S. Pond, 1971: The determination of the Kolmogoroff constants for velocity, temperature and humidity fluctuations from second and third order structure functions. J. Fllid Mech., 50, 257-269.

Phillips, O. M., 1966: The Dynamics of the Upper Ocean. Cambridge University Press, $261 \mathrm{pp}$.
- - 1971: On spectra measured in an undulating layered medium. J. Plys. Oceanogr., 1, 1-6.

Roden, G. I., 1971: Spectra of North Pacific temperature and salinity perturbations in the depth domain. J. Phys. Oceanogr., 1, 25-33.

Rosenblatt, M., and J. W. Van Ness, 1965: Estimation of the bispectrum. Ann. Math. Statist., 36, 1120-1136.

Tukey, J. W., 1965 : Data analysis and the frontiers of geophysics. Science, 148, 1283-1289.

Van Atta, C. W., and W. Y. Chen, 1970: Structure functions of turbulence in the atmospheric boundary layer over the ocean. J. Fluid Mech., 44, 145-159.

Vigoureux, P., and J. B. Hersey, 1962: Sound in the sea. The Sea, Vol. 1, New York, Interscience, 476-497. 